\title{
Salt-induced collapse and reexpansion of highly charged flexible polyelectrolytes
}

\author{
Pai-Yi Hsiao and Erik Luijten* \\ Department of Materials Science and Engineering and Frederick Seitz Materials Research Laboratory, \\ University of Illinois at Urbana-Champaign, Urbana, Illinois 61801, U.S.A.
}

(Dated: May 22, 2006)

\begin{abstract}
We study the salt-dependent conformations of dilute flexible polyelectrolytes in solution via computer simulations. Low concentrations of multivalent salt induce the known conformational collapse of individual polyelectrolyte chains, but as the salt concentration is increased further this is followed by a reexpansion. We explicitly demonstrate that multivalent counterions can overcompensate the bare charge of the chain in the reexpansion regime. Both the degree of reexpansion and the occurrence of overcharging sensitively depend on ion size. Our findings are relevant for a wide range of salt-induced complexation phenomena.

PACS numbers: 82.35.Rs, 36.20.Ey, 87.15.He, 87.15.Aa
\end{abstract}

Many biological and synthetic polyelectrolytes undergo two macroscopic phase transitions upon addition of multivalent salt or charged small molecules [1, 2, 3, 4, [5]. First, precipitation of polyelectrolytes occurs if the concentration of the added salt exceeds a critical value. This precipitate subsequently redissolves if the concentration is increased beyond a second critical value. These phenomena, jointly referred to as reentrant condensation [6], have attracted considerable attention because they are a fundamental and generic aspect of polyelectrolyte behavior, with potential relevance for the understanding and development of biological phenomena and applications such as gene delivery [7]. Under dilute conditions, the formation and redissolution of multimolecular aggregates are replaced by, respectively, single-chain "collapse" and reexpansion. This behavior is directly observed in, e.g., the stretching of individual DNA coils in salty solution [8].

Whereas several theoretical explanations for reentrant condensation have been proposed [6, 9, 10, 11], important open questions remain regarding the suggested mechanisms, and several predictions have only partially been verified. Two main scenarios can be distinguished. (1) Charge inversion: Counterions form a strongly correlated liquid at the polyelectrolyte surface and, at high salt concentration, overcompensate the bare chain charge. Condensation of a polyelectrolyte is predicted to take place if its effective charge is close to zero [6, 12]. (2) The two-state model [9] explains precipitation and redissolution of flexible polyelectrolytes by assuming that individual chains adopt either a collapsed or an extended state. This scenario predicts a strong sensitivity to ion size, which is not considered in Refs. 6, 12]. In addition, in this case overcharging does not occur by necessity, but may be mitigated by coion association [1].

Computer simulations offer the possibility to study this behavior at a microscopic level, but hitherto most simulations of flexible chains have focused on solutions with only divalent salt 13, 14] or no salt at all [15, 16, 17, 18]. Whereas chain collapse has been observed under the in- fluence of divalent salt or counterions [16], the reexpansion observed in experiments and predicted by theory has not been reproduced in computer simulations. In this Letter, we aim to clarify this apparent discrepancy and to understand the behavior of strong polyelectrolytes in solutions of multivalent salt by studying the conformations of dilute chains over a wide range of salt concentrations. Our calculations allow us to explicitly address the theoretical predictions. Specifically, we answer the following questions: (i) How does the conformation of a polyelectrolyte depend on salt concentration and valency, and under what conditions can an extended (i.e., noncollapsed) structure be observed at high concentrations? (ii) Is there a dependence on ion size, as suggested by some theories? (iii) Can the charge of a polyelectrolyte be overcompensated by condensed ions, such that its effective charge is reversed compared to the bare charge?

Following Stevens and Kremer [15], we model an anionic polyelectrolyte as a bead-spring model in a continuous medium of uniform dielectric constant $\varepsilon$, representing the solvent. A chain consists of $N$ monomers of size $\sigma$, which each carry a charge $-e$. Adjacent monomers are connected by a FENE bond potential 15] with maximum extension $R_{0}=2 \sigma$ and spring constant $k=7 \varepsilon_{\mathrm{LJ}} / \sigma^{2}$. The counterions are monovalent, whereas the added salt dissociates into $Z$-valent cations and monovalent anions. Electrostatic interactions are computed via the Ewald summation and the excluded volume of monomers and ions is modeled via the shifted-truncated Lennard-Jones potential with coupling constant $\varepsilon_{\mathrm{LJ}}=k_{\mathrm{B}} T / 1.2$, where $k_{\mathrm{B}}$ denotes Boltzmann's constant and $T$ the absolute temperature. Periodic boundaries are imposed. We express the Coulomb interaction between particles $i$ and $j$ as $k_{\mathrm{B}} T \lambda_{\mathrm{B}} z_{i} z_{j} / r$, where $\lambda_{\mathrm{B}} \equiv e^{2} /\left(4 \pi \varepsilon \varepsilon_{0} k_{\mathrm{B}} T\right)$ is the Bjerrum length, $\varepsilon_{0}$ the vacuum permittivity, and $z_{i}$ and $z_{j}$ the valencies of the particles. In aqueous solution at room temperature, $\lambda_{\mathrm{B}}=7.14 \AA$. We set $\lambda_{\mathrm{B}}=3 \sigma$, representative of a prototypical flexible polyelectrolyte such as sodium poly(styrene sulfonate).

To compute conformational properties, we employ 


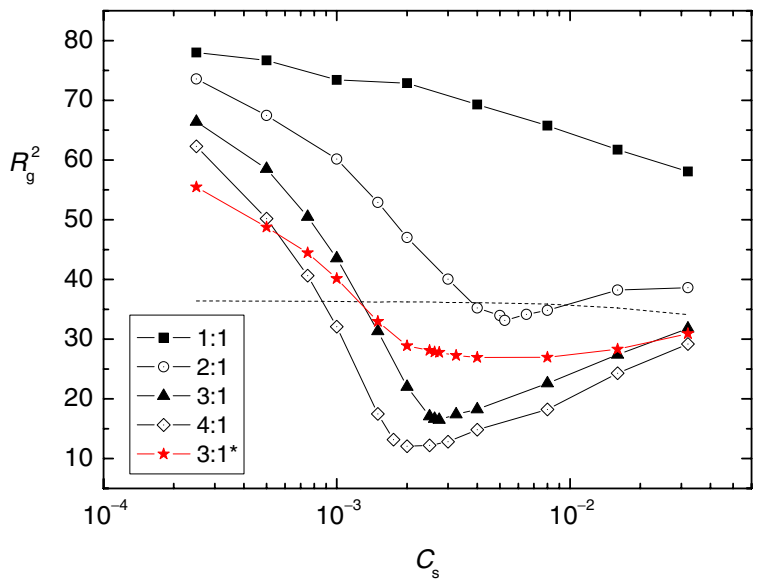

FIG. 1: Squared radius of gyration $R_{g}^{2}$ of a polyelectrolyte of length $N=64$ as a function of salt concentration $C_{s}$. Curves corresponds to added salt with counterion valency from 1 to 4 . The curve $3: 1^{*}$ refers to a system with ions that have a twice smaller diameter (see text for details). The dashed line corresponds to a reference system without any electrostatic interactions. Error bars are smaller than the symbol size.

Monte Carlo simulations, which makes it possible to accelerate the relaxation of longer chains via reptation moves. Since we focus on the dilute polyelectrolyte regime, relatively large simulation cells are required, resulting in considerable ion numbers in systems with added salt. For computational efficiency, we therefore confine ourselves to a monomer concentration $C_{m}=0.008 \sigma^{-3}$, which corresponds to $0.99 \mathrm{~mol} / \ell$. This is higher than typical experimental conditions [1], but we have explicitly verified that our findings are representative for the entire concentration range $0.001 \sigma^{-3} \leq$ $C_{m} \leq 0.008 \sigma^{-3}$. In addition, for all chain lengths studied here $(N=16,32,64,96), C_{m}$ lies well below the overlap threshold $C_{m}^{*}$ as estimated from the radius of gyration $R_{g}$. We adopt $\sigma, \sigma^{-3}$, and $e$ as our units of length, concentration, and charge, respectively.

We first study the conformation of a polyelectrolyte of length $N=64$ in the presence of $(Z: 1)$-salt, where $Z$ is varied from 1 to 4 . Ions and monomers have the same size. Figure 1 shows the average squared radius of gyration $R_{g}^{2}$ as a function of salt concentration. In the absence of salt, the chain adopts an extended conformation, owing to the electrostatic repulsions between monomers. Upon addition of monovalent salt, these repulsions are screened and $R_{g}^{2}$ gradually decreases. By contrast, for multivalent salt, a much stronger decrease in $R_{g}^{2}$ is seen, occurring at considerably lower salt concentrations. This is the conformational collapse that has previously been observed for polyelectrolytes with multivalent counterions under salt-free conditions [14, 16]. The smallest value of $R_{g}^{2}$ occurs for $C_{s}$ near $C_{Z}$, the $(Z: 1)$-salt concentration at which the total charge of the $Z$-valent cations neutralizes the bare polyelectrolyte charge. Accordingly, this compact state occurs at a salt concentration that decreases with increasing valency $Z$, consistent with the two-state model [9, 10]. In addition, higher counterion valency leads to more compact states. A striking effect occurs once the salt concentration is increased beyond $C_{Z}$. The chain starts to swell again, in close analogy with the redissolution observed for multichain aggregates [1, 2]. Although the chains that are collapsed by trivalent and tetravalent counterions reexpand strongly, they remain more compact than the chain in 2:1 salt for all salt concentrations investigated. Comparison with the structure of the same polyelectrolyte in monovalent salt, which exhibits a slow, monotonic decrease of $R_{g}^{2}$ with increasing $C_{s}$, emphasizes the important role of counterion valency. The salt concentration at which the reexpanded state is reached is insensitive to monomer concentration, whereas the collapse concentration $C_{Z}$ is proportional to $C_{m}$. Thus, our relatively high choice of $C_{m}$ causes both conformational changes to occur over a narrower range in salt concentration than in typical experiments.

We further quantify the swelling of the polyelectrolyte coil by investigating the variation of $R_{g}$ with degree of polymerization $N$, for tetravalent salt. As anticipated from scaling theory [19], the radius of gyration is described by a power law $N^{\nu}$ for the range of chain lengths studied here $(16 \leq N \leq 96)$. Figure 2 depicts the concentration-dependent exponent $\nu$ extracted from least-squares fits at fixed $C_{s}$. At low concentrations, $\nu$ exceeds the self-avoiding random-walk value $\nu_{\mathrm{SAW}}=0.588$, owing to the electrostatic repulsions be-

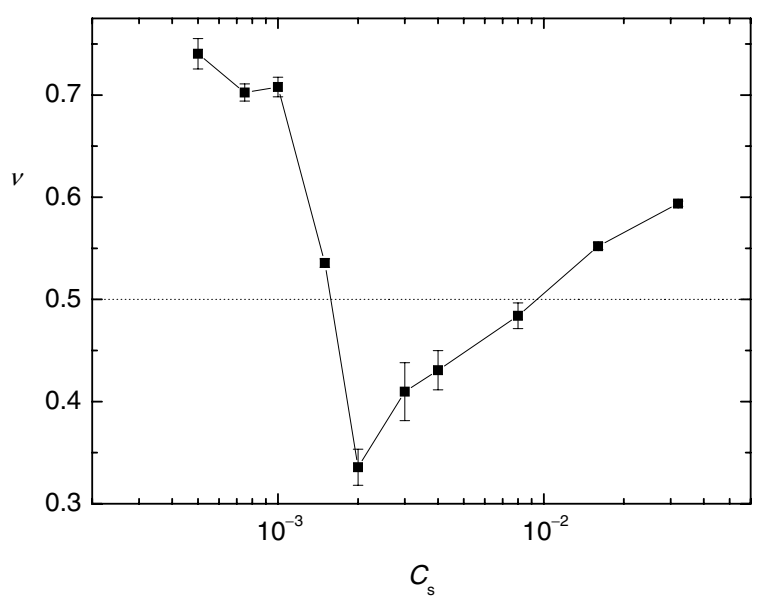

FIG. 2: Scaling dimension $\nu$ of the radius of gyration for a polyelectrolyte in a tetravalent salt solution. At low salt concentration $C_{s}, \nu$ exceeds the self-avoiding random-walk value 0.588 , owing to the electrostatic repulsions between monomers. For the concentration $C_{Z=4}$ corresponding to the minimum in Fig. 11 the exponent reaches the value for a compact globule. The subsequent reexpansion of the chain at higher salt concentrations is reflected in an increase in $\nu$. 


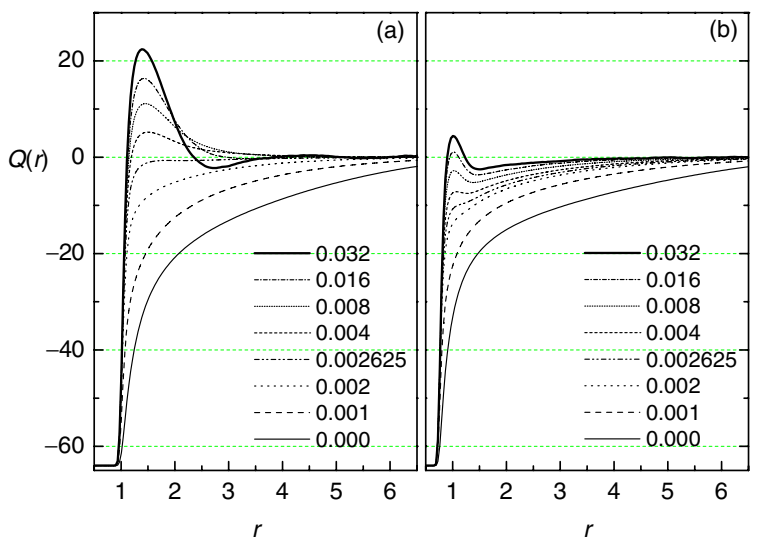

FIG. 3: Net charge $Q(r)$ within a tube of radius $r$ around a chain $(N=64)$ at various salt concentrations $C_{s}$ of trivalent salt. (a) For ion diameter $\sigma$ (case 3:1 in Fig. 1) an overcharging peak appears at short $r$. (b) For smaller ions (case 3:1*) the overcharging peak is absent.

tween monomers [20]. Near the neutralization concentration $C_{Z=4}=0.002$, the chain attains its most compact state and $\nu=0.34 \pm 0.02$, in good agreement with the poor-solvent value, $\nu=\frac{1}{3}$. Upon reexpansion, the exponent $\nu$ increases and approximately reaches $\nu_{\mathrm{SAW}}$ at the highest concentrations.

Chain reexpansion has been linked to charge reversal (or overcharging) 12]. Since for spherical colloids overcharging was found to strongly depend on ion excluded volume [21], we proceed to clarify the effect of ion size in the case of flexible polyelectrolytes. To this end, we repeat our calculations for 3:1 salt in which all ions have a diameter $\sigma / 2$, i.e., twice smaller than the monomers. The electrostatic attraction between a trivalent counterion and a monomer at contact thus increases by a factor $\frac{4}{3}$ and equals the attraction experienced by tetravalent ions of diameter $\sigma$. As the smaller ions allow the chain to adopt a more compact structure, it may appear plausible that in this situation $R_{g}$ is even smaller than for tetravalent salt and hence also smaller than $R_{g}$ for trivalent salt of diameter $\sigma$. However, as shown in Fig. [1 (cf. the curve labeled $3: 1^{*}$ ), the actual situation is more complicated. The polyelectrolyte chain is indeed less extended than in tetravalent salt solution for low salt concentrations and shrinks further if $C_{s}$ is increased. But this decrease is weaker than for larger ions, and near the neutralization condition the chain is much less compact than for tetravalent or even trivalent salt of diameter $\sigma$. For higher salt concentrations we observe a gradual expansion of the chain, and $R_{g}$ reaches a magnitude that is approximately the same for both trivalent cases.

The mechanism for this remarkable dependence on ion size can be understood from the charge distribution around the polyelectrolyte. We define a worm-like tube around the chain, consisting of $N$ spheres of ra- dius $r$ centered around the monomers, and monitor the ensemble-averaged net charge $Q(r)$ within this tube, as a function of salt concentration. As shown in Fig. B(a) for trivalent salt with ion size $\sigma, Q(r)$ is always negative for $0 \leq C_{s}<C_{Z=3}=0.00267$ and only approaches zero at large $r$, to satisfy electroneutrality. In this regime, upon addition of salt all trivalent counterions condense on the chain and replace the monovalent counterions, as predicted [9]. As $C_{s}$ approaches $C_{Z=3}$ the magnitude of the net charge $Q(r)$ decreases, until the chain is completely neutralized by the condensed trivalent counterions and takes a compact structure. For $C_{s}>C_{Z=3}$, not all trivalent ions condense, although the number of condensed trivalent counterions can exceed the number required to neutralize the chain, in accordance with Refs. 6, [12]. This overcharging is reflected in the appearance of a strong peak at small tube diameter $r$ in Fig. 31(a). The excess charge attracts coions that form a second layer, resulting in a rapid decrease of the peak with increasing $r$. At the highest salt concentration investigated, $Q(r)$ exhibits an oscillatory tail, indicative of a multilayered ionic structure, similar to what has been observed for electrical double layers 22], colloids 23] and rod-like polyelectrolytes 24]. Interestingly, for monovalent salt (not shown) no overcharging peak occurs, consistent with the absence of important conformational changes when the salt concentration is varied.

The behavior of $Q(r)$ drastically changes upon reduction of the ion size, as illustrated in Fig. 3(b) for case $3: 1^{*}$ (ion diameter $\sigma / 2$ ). Again, the net charge within the tube decreases with increasing salt concentration, but it is not neutralized at $C_{Z=3}$, consistent with a much larger coil size than for the system with larger ions (Fig. 11). For $C_{s}>C_{Z=3}$ a small peak appears in $Q(r)$, but there is hardly any overcharging even at the highest concentrations. A study of the ion configurations in the simulations reveals that this remarkable difference arises from the strong increase in counterion-coion attraction if their size is reduced. This association, akin to the formation of Bjerrum pairs, reduces the effective valency of the salt. Consequently, compared to the 3:1 case, the chain exhibits a less strong conformational collapse and $Q(r)$ remains more negative. When pairs of trivalent counterions and monovalent coions condense on the chain, they tend to be oriented with the trivalent ion toward the monomer, explaining the appearance of a small peak in $Q(r)$. Analogous behavior has been observed for simple colloids [21], indicating that the decrease in overcharging upon reduction of ion size is a generic effect. It also explains why neither reexpansion nor overcharging of a polyelectrolyte were observed in earlier simulations of a flexible chain in the presence of divalent salt 14. In those simulations the ion sizes were chosen as half the monomer size 25].

It is tempting to assign an "effective" charge to the chain based upon the net tube charge $Q(r)$. However, as Fig. [3 indicates, such an interpretation is arbitrary if 


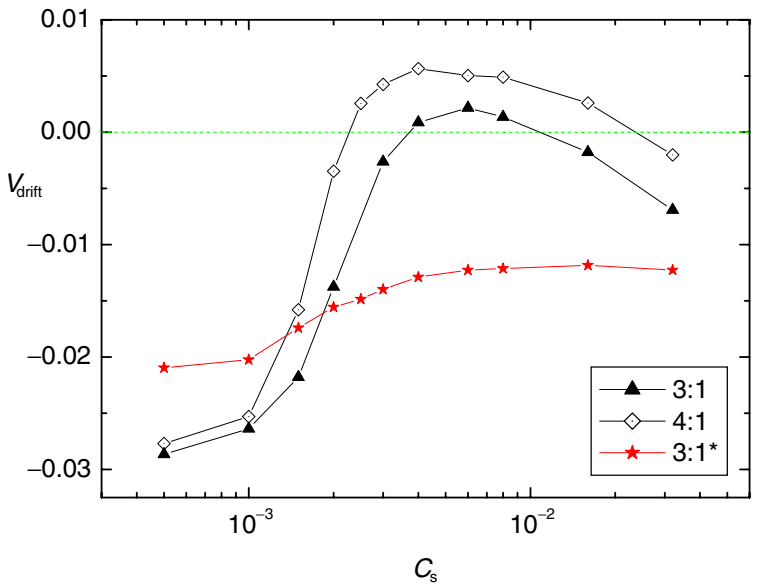

FIG. 4: Drift velocity $V_{\text {drift }}$ (in units of $\sigma / \tau$ ) of the center of mass of a polyelectrolyte $(N=16)$ as a function of salt concentration $C_{s}$, in the direction of the electric field. The sign reversal in the electrophoretic mobility provides direct evidence for charge reversal by multivalent counterions.

there is no clear prescription for the proper choice of the tube radius. To circumvent this ambiguity, we study the electrophoretic mobility as a function of salt valency and concentration for a polyelectrolyte of $N=16$ monomers via Langevin dynamics simulations, using the LAMMPS package 26]. The collision frequency in the thermostat equals $\gamma=\tau^{-1}$, where $\tau=\sigma \sqrt{m /\left(k_{\mathrm{B}} T\right)}$ is the time unit and $m$ the ion mass. We apply a constant electric field of magnitude $E=0.1 k_{\mathrm{B}} T /(e \sigma)$, which, as we carefully verified, does not distort the conformational properties of the chain or the charge distribution around it 27]. Due to the absence of hydrodynamic effects this calculation does not realistically reproduce dynamic properties, but it will properly reflect the sign of the electrophoretic mobility and can thus be used to probe the effective charge without imposing an arbitrary criterion for the condensation of specific ions. Indeed, the center-of-mass drift velocity of the chain (Fig. 4) reveals a striking dependence on salt concentration. For both trivalent and tetravalent salt, it exhibits a zero crossing, i.e., the electrophoretic mobility of the chain changes sign upon addition of salt, as observed experimentally for colloids 28. This sign reversal occurs close to the neutralization condition (minimum in Fig. (1) and thus depends on counterion valency. Furthermore, for trivalent salt with smaller ion size (case $\left.3: 1^{*}\right)$ no sign reversal occurs, i.e., no overcharging takes place. We view these observations as a strong confirmation of the charge reversal suggested by Fig. 3 and as evidence for the relation between charge reversal and reexpansion, but also note that a less strong collapse and subsequent reexpansion can occur without overcharging. At the highest salt concentrations, a second sign reversal occurs, which is possibly related to the formation of a multi-layered structure of counterions and coions, consistent with the oscillatory behavior in Fig. 3 [27].

In summary, we have demonstrated, for the first time, that dilute flexible polyelectrolytes in a solution with multivalent salt undergo not only a conformational collapse, but also a reexpansion. These findings support the two-state model for polyelectrolyte conformations [9, 10] and confirm the important role of ion excluded volume 11]. Furthermore, we have presented unambiguous evidence for overcharging by means of condensed multivalent counterions at high salt concentrations 6,12 , in which the reversal of the effective charge coincides with the most compact chain conformation.

This material is based upon work supported by the U.S. Department of Energy, Division of Materials Sciences under Grant No. DEFG02-91ER45439, through the Frederick Seitz Materials Research Laboratory at the University of Illinois at Urbana-Champaign.

* Corresponding author. E-mail: luijten@uiuc.edu

[1] M. Olvera de la Cruz, L. Belloni, M. Delsanti, J. P. Dalbiez, O. Spalla, and M. Drifford, J. Chem. Phys. 103, 5781 (1995).

[2] J. Pelta, F. Livolant, and J.-L. Sikorav, J. Biol. Chem. 271, 5656 (1996).

[3] E. Raspaud, M. Olvera de la Cruz, J.-L. Sikorav, and F. Livolant, Biophys. J. 74, 381 (1998).

[4] I. Sabbagh, M. Delsanti, and P. Lesieur, Eur. Phys. J. B 12, 253 (1999).

[5] L. K. Sanders, C. Guáqueta, T. E. Angelini, J.-W. Lee, S. C. Slimmer, E. Luijten, and G. C. L. Wong, Phys. Rev. Lett. 95, 108302 (2005).

[6] T. T. Nguyen, I. Rouzina, and B. I. Shklovskii, J. Chem. Phys. 112, 2562 (2000).

[7] V. Vijayanathan, T. Thomas, and T. J. Thomas, Biochemistry 41, 14085 (2002).

[8] Y. Murayama, Y. Sakamaki, and M. Sano, Phys. Rev. Lett. 90, 018102 (2003).

[9] F. J. Solis and M. Olvera de la Cruz, J. Chem. Phys. 112, 2030 (2000).

[10] F. J. Solis and M. Olvera de la Cruz, Eur. Phys. J. E 4, 143 (2001).

[11] F. J. Solis, J. Chem. Phys. 117, 9009 (2002).

[12] A. Y. Grosberg, T. T. Nguyen, and B. I. Shklovskii, Rev. Mod. Phys. 74, 329 (2002).

[13] M. J. Stevens and S. J. Plimpton, Eur. Phys. J. B 2, 341 (1998).

[14] S. Liu, K. Ghosh, and M. Muthukumar, J. Chem. Phys. 119, 1813 (2003).

[15] M. J. Stevens and K. Kremer, J. Chem. Phys. 103, 1669 (1995).

[16] R. G. Winkler, M. Gold, and P. Reineker, Phys. Rev. Lett. 80, 3731 (1998).

[17] M. J. Stevens, Biophys. J. 80, 130 (2001).

[18] H. J. Limbach and C. Holm, J. Phys. Chem. B 107, 8041 (2003).

[19] P. Pfeuty, R. M. Velasco, and P. G. de Gennes, J. Phys. (Paris) Lett. 38, 5 (1977).

[20] The effective value of $\nu$ in the absence of salt sensitively 
depends on the monomer concentration.

[21] R. Messina, E. González-Tovar, M. Lozada-Cassou, and C. Holm, Europhys. Lett. 60, 383 (2002).

[22] H. Greberg and R. Kjellander, J. Chem. Phys. 108, 2940 (1998).

[23] T. Terao and T. Nakayama, Phys. Rev. E 63, 041401 (2001).

[24] M. Deserno and C. Holm, Mol. Phys. 100, 2941 (2002).

[25] For the conditions examined here, this size would be un- realistically small, if one takes into account hydration of the ions.

[26] S. J. Plimpton, J. Comp. Phys. 117, 1 (1995).

[27] P.-Y. Hsiao and E. Luijten, to be published.

[28] A. Martin-Molina, M. Quesada-Pérez, F. GalisteoGonzález, and R. Hidalgo-Álvarez, J. Chem. Phys. 118, 4183 (2003). 